\title{
Expression of cancer stem cells markers in urinary bladder urothelial carcinoma and its precursor lesions
}

\author{
Jaromir Hacek ${ }^{\mathrm{a}}$, Antonin Brisuda ${ }^{\mathrm{b}}$, Marek Babjuk ${ }^{\mathrm{b}}$, Josef Zamecnik ${ }^{\mathrm{a}}$
}

\begin{abstract}
Background. Cancer stem cells (CSC) and their role in tumorigenesis of various solid tumors have been studied in past decades. Urothelial CSC were first identified 10 years ago and subsequent studies have been performed with the aim to identify reliable markers of CSC. So far, a few studies have investigated a relationship between CSC markers expression in urothelial carcinoma tissue and histopathological characteristics of the tumor.

Methods. In our study, we evaluated an immunoexpression of the CSC markers CD24, CD44, CD66 and CD133 in tissue sections of urothelial carcinoma (all tumor grades and stages were included), urothelial carcinoma in situ and non-neoplastic urothelium, totally 218 specimens were enrolled.

Results. All studied molecules were expressed either in tumor tissue and non-neoplastic urothelium. Urothelial carcinomas of higher tumor grade and stage expressed molecules CD24 and CD133 significantly more frequently whereas molecules CD44 and CD66 did not show significant association with tumor histopathological features.

Conclusions. Our results showed that studied molecules are not suitable for direct detection of CSC in urothelial carcinoma tissue sections, but an expression of molecules CD24 and CD133 is significantly related to urothelial carcinoma grade and stage, which are both important prognostic indicators and therefore an expression of these markers might have a potential prognostic value.
\end{abstract}

Key words: cancer stem cells, immunohistochemistry, markers, urothelial carcinoma

Received: January 24, 2020; Revised: April 6, 2020; Accepted: April 6, 2020; Available online: May 18, 2020 https://doi.org/10.5507/bp.2020.017

(c) 2021 The Authors; https://creativecommons.org/licenses/by/4.0/

${ }^{a}$ Department of Pathology and Molecular Medicine, Second Faculty of Medicine, Charles University and Motol University Hospital, Prague, Czech Republic

${ }^{b}$ Department of Urology, Second Faculty of Medicine, Charles University and Motol University Hospital, Prague, Czech Republic Corresponding author: Jaromir Hacek, e-mail:jaromir.hacek@fnmotol.cz

\section{INTRODUCTION}

Urinary bladder cancer is the $7^{\text {th }}$ most common cancer worldwide with the highest incidence rates in Western Europe, North America and Australia ${ }^{1}$. The most common type of bladder cancer is urothelial carcinoma (UC), which represents a significant cause of morbidity and mortality ${ }^{2}$. The majority of UC are non-invasive papillary tumors with high recurrence rate and low (10-20\%) risk of progression into invasive tumor during recurrences. Only approximately $20 \%$ of UC are primarily muscleinvasive tumors with aggressive behavior ${ }^{1}$.

In the past decades, a concept of cancer stem cells (CSC) was developed ${ }^{3,4}$. Opposite to stochastic theory postulating that all tumor cells have an equal potential of proliferation and tumor initiation, the CSC model holds that cancer cells are hierarchically organized and at the apex of the hierarchy is a small subpopulation of cells with features of non-neoplastic stem cells and with ability of self-renewal that are responsible for tumor initiation and progression and due to their resistance to chemotherapy and radiotherapy also for tumor recurrence ${ }^{5}$.

Many studies have been focused on identification and isolation of CSC and their markers both in vitro and in vivo in various tumors. These studies use stem cell enrichment by morphological and drug selection, cell sorting via surface markers or gene expression. Properties of CSC are tested through functional assays (e.g. hematopoietic stem cell assay, colony formation and sphere formation assay) $\left(\right.$ ref. $\left.^{5}\right)$. The CSC model was first tested and confirmed in hematologic malignancies and later also in solid tumors such as breast, lung, pancreatic, colon, ovarian or prostate cancer ${ }^{6}$.

UC CSC were first identified in 2009 and subsequent studies were performed with an effort to discover reliable markers of CSC. CD24, CD44, CD66 and CD133 molecules are included among well accepted CSC markers in $\mathrm{UC}\left(\right.$ ref. $\left.^{7-11}\right)$, similarly to other solid cancers.

CD44 is considered a potential CSC marker in the vast majority of neoplasms. It is a transmembrane glycoprotein associated with many other proteins responsible for cell adhesion, proliferation, survival or migration ${ }^{7,9}$. CD24 is a surface protein functioning in intercellular and cell-matrix interaction. It is involved in the cell adhesion and the process of metastasis ${ }^{7,9}$. CD66 (CEACAM6) is an adhesion molecule expressed in the junction with the tumor stroma ${ }^{12}$. CD133 (PROM1) is a transmembrane glycoprotein often expressed on adult stem cells maintaining stem cell properties by suppressing differentiation ${ }^{10,12}$.

There are only a few studies dealing with the immunohistochemical expression of CSC markers in human bladder UC tumor tissue $\mathrm{e}^{7,11,13,14}$. In our study, we investigated 
the immunohistochemical expression of molecules CD24, CD44, CD66 and CD133 in urothelial carcinomas as well as in precancerous lesions of the urinary bladder, and we analyzed its potential relationship with histopathological and prognostic parameters of the disease.

\section{MATERIALS AND METHODS}

\section{Patient characteristics and material used}

Tissue samples from 137 patients (113 males, 24 females), age range from 11 to 88 years (mean, 67.0), were included in our retrospective study.

All patients underwent a surgical procedure - transurethral biopsy, transurethral resection or radical cystectomy. In 81 patients more than one type of histological urothelial lesion was revealed in cystectomy specimen. In total, 218 histopathologically defined urothelial lesions were enrolled including primary UC of urinary bladder (both superficial - stages Ta, T1 and muscle invasive - stages T2, T3 and T4 according to TNM classification ${ }^{15}$ ), urothelial carcinoma in situ (CIS) or non-neoplastic urothelium (see Table 1). Urothelial carcinomas in situ were solitary in 13 cases and 18 were associated with UC. Histologically normal urothelium was available in 63 cystectomy specimens and it was taken from areas not adjacent to the urothelial tumor; 11 samples of normal urothelium was obtained from transurethral biopsies in patients without urothelial tumor.

Archived formalin-fixed and paraffin-embedded tissues were used to prepare hematoxylin and eosin (HE) stained slides and serial sections contiguous to those used for histology were used for further immunohistochemical analysis. All cases were first reviewed to confirm the histological type of the lesion, and tumor histopathological parameters including tumor grade according to the World Health Organisation 1973 and 2004/2016 classification ${ }^{1}$, tumor stage according to UICC TNM Classification (8th ed.) (ref. ${ }^{15}$ ) and lymph node status in cases of invasive tumors. Detailed information concerning the proportion of specimen types and their histopathologic characteristics is given in Table 1.

\section{Immunohistochemistry}

Immunohistochemistry staining was performed on 4 $\mu \mathrm{m}$ thick tissue sections which were deparaffinized and rehydrated. Heat-induced epitope retrieval was performed in sodium citrate buffer solution ( $\mathrm{pH} \mathrm{6.0)}$ warming up to $96{ }^{\circ} \mathrm{C}$ in water bath for $40 \mathrm{~min}$. Following cooling for $20 \mathrm{~min}$ and blocking of endogenous peroxidase activity, sections were incubated overnight at $4{ }^{\circ} \mathrm{C}$ with following primary antibodies: CD24 (mouse monoclonal, anti-human, Abcam, USA; dilution 1:300), CD44 (mouse monoclonal, anti-human, LifeSpan CA, USA; dilution 1:500), CD66 (mouse monoclonal, anti-human, Exbio, CZ; dilution 1:200), CD133 (mouse monoclonal, anti-human, Biorbyt, UK; dilution 1:200). After washing with PBS buffer sections were incubated for 20 min with Mouse/Rabbit PolyDetector HRP with DAB (BioSB, Santa Barbara, CA,
Table 1. Patient and specimens characteristics.

\begin{tabular}{|c|c|}
\hline $\begin{array}{l}\text { Number of patients } \\
\text { Age range } \\
\text { Mean age }\end{array}$ & $\begin{array}{c}137 \\
11-88 \\
67.0\end{array}$ \\
\hline \multicolumn{2}{|l|}{ Gender } \\
\hline Male & $113(82.5 \%)$ \\
\hline Female & $24(17.5 \%)$ \\
\hline \multicolumn{2}{|l|}{ Type of lesion } \\
\hline Total & $218(100 \%)$ \\
\hline Normal urothelium & $74(33.9 \%)$ \\
\hline Urothelial carcinoma in situ (UCIS) & $32(14.7 \%)$ \\
\hline Urothelial carcinoma (UC) & $112(51.4 \%)$ \\
\hline \multicolumn{2}{|l|}{ Grade (WHO 2016) } \\
\hline Total (UC) & $112(100 \%)$ \\
\hline Low grade & $35(31.3 \%)$ \\
\hline High grade & $77(68.7 \%)$ \\
\hline \multicolumn{2}{|l|}{ Grade (WHO 1973) } \\
\hline Total (UC) & $112(100 \%)$ \\
\hline Grade 1 & $7(6.2 \%)$ \\
\hline Grade 2 & $30(26.8 \%)$ \\
\hline Grade 3 & $75(67.0 \%)$ \\
\hline \multicolumn{2}{|l|}{ Stage } \\
\hline Total (UC) & $112(100 \%)$ \\
\hline $\mathrm{Ta}$ & $37(33.0 \%)$ \\
\hline $\mathrm{T} 1$ & $16(14.3 \%)$ \\
\hline $\mathrm{T} 2$ & $16(14.3 \%)$ \\
\hline $\mathrm{T} 3$ & $26(23.2 \%)$ \\
\hline $\mathrm{T} 4$ & $17(15.2 \%)$ \\
\hline \multicolumn{2}{|l|}{ Clinical stage } \\
\hline Total (UC and UCIS) & $144(100 \%)$ \\
\hline Non-muscle-invasive $(\mathrm{Ta}+\mathrm{T} 1)$ & $53(36.8 \%)$ \\
\hline Muscle-invasive (T2+T3+T4) & $59(41.0 \%)$ \\
\hline Carcinoma in situ (Tis) & $32(22.2 \%)$ \\
\hline \multicolumn{2}{|l|}{ Lymph node status } \\
\hline Total (Invasive urothelial carcinoma) & $75(100 \%)$ \\
\hline No & $60(80.0 \%)$ \\
\hline $\mathrm{N} 1+\mathrm{N} 2$ & $15(20.0 \%)$ \\
\hline
\end{tabular}

UC - Urothelial Carcinoma, UCIS - Urothelial Carcinoma In Situ

USA). Slides were counterstained with Harris's hematoxylin, dehydrated and mounted.

Sections stained with anti-CD24, CD44, CD66 and CD133 antibodies were evaluated by two experienced pathologists blinded to clinicopathological data.

Expression of all the above mentioned markers was evaluated in 10 representative high power fields (HPF, $\mathrm{x} 400$ ) and percentages of positive cells were calculated. Only cytoplasmic and membrane immunostaining of intensity at least 1 (scored semi-quantitatively as follows: 0 - negative, 1 - weak, 2 - moderate, 3 - strong) of each marker was regarded as positive. Cases with at least one positive epithelial tumor cell per HPF were classified as positive for marker expression. Distribution/pattern of the immunopositivity in the lesion was also noted.

\section{Statistics}

Statistical analysis was performed using R v. 3.0.2 software. Association between clinicopathological parameters 
Table 2. Association of CD24 and CD133 expression with the histopathological characteristics of urinary bladder UC.

\begin{tabular}{|c|c|c|c|c|c|}
\hline & total number & CD 24 positive & $P$ & CD 133 positive & $P$ \\
\hline Grade (WHO 1973) & & & $<0.0001$ & & 0.0032 \\
\hline Total (UC) & 112 & $72(64.3 \%)$ & & $47(42.0 \%)$ & \\
\hline Grade 1 & 7 & $1(14.3 \%)$ & & $0(0.0 \%)$ & \\
\hline Grade 2 & 30 & $4(13.3 \%)$ & & $3(10.0 \%)$ & \\
\hline Grade 3 & 75 & $67(89.3 \%)$ & & $44(58.7 \%)$ & \\
\hline Grade (WHO 2016) & & & $<0.0001$ & & $<0.0001$ \\
\hline Total (UC) & 112 & $72(64.3 \%)$ & & $47(42.0 \%)$ & \\
\hline Low-grade & 35 & $4(11.4 \%)$ & & $2(5.7 \%)$ & \\
\hline High-grade & 77 & $68(88.3 \%)$ & & $45(58.4 \%)$ & \\
\hline Stage classification & & & $<0.0001$ & & 0.0001 \\
\hline Total (UC) & 112 & $72(64.3 \%)$ & & $47(42.0 \%)$ & \\
\hline $\mathrm{Ta}$ & 37 & $4(10.8 \%)$ & & $4(10.8 \%)$ & \\
\hline $\mathrm{T} 1$ & 16 & $13(81.3 \%)$ & & $6(37.5 \%)$ & \\
\hline $\mathrm{T} 2$ & 16 & $13(81.3 \%)$ & & $11(68.8 \%)$ & \\
\hline T3 & 26 & $25(96.2 \%)$ & & $16(61.5 \%)$ & \\
\hline $\mathrm{T} 4$ & 17 & $17(100.0 \%)$ & & $10(58.8 \%)$ & \\
\hline Depth of invasion & & & $<0.0001$ & & $<0.0001$ \\
\hline Total (UC) & 112 & $72(64.3 \%)$ & & $47(42.0 \%)$ & \\
\hline Non-muscle-invasive $(\mathrm{Ta}+\mathrm{T} 1)$ & 53 & $17(32.1 \%)$ & & $10(18.9 \%)$ & \\
\hline Muscle-invasive (T2+T3+T4) & 59 & $55(93.2 \%)$ & & $37(62.7 \%)$ & \\
\hline Lymph node status & & & 1.000 & & 0.2438 \\
\hline Total (invasive UC) & 75 & $68(90.7 \%)$ & & $43(57.3 \%)$ & \\
\hline N0 & 60 & $54(90.0 \%)$ & & $32(53.3 \%)$ & \\
\hline $\mathrm{N} 1+\mathrm{N} 2$ & 15 & $14(93.3 \%)$ & & $11(73.3 \%)$ & \\
\hline
\end{tabular}

UC - Urothelial Carcinoma

and expression of the studied markers was evaluated using Fisher's exact test. $P<0.05$ was considered as statistically significant difference.

\section{RESULTS}

\section{Urothelial Carcinoma}

Immunoexpression of all the studied markers (CD24, CD44, CD66 and CD133) was localized both in membranes and in cytoplasm of epithelial tumor cells, surrounding stroma was negative. There were no obvious morphological differences between the positive and the negative tumor cells. The positivities were either diffuse in neoplastic urothelium (Fig. 1.) or only focal (Fig. 2.) (Table 2 and 3). Single immunopositive cells or small groups of tumor cells were localized dispersely throughout the neoplastic urothelium, both in the superficial and in the deep invasive component of the tumor.

Markers CD24 and CD133 were significantly more often expressed in carcinomas with both higher grade and higher stage (Table 2). No significant association among expression of CD44 and CD66 and histopatho- logical parameters of UC was observed in our study group (Table 3).

\section{Urothelial CIS}

CSC markers expression was evaluated in 32 cases of urothelial CIS. CD24 and CD 133 showed diffuse membranous and cytoplasmic positivity in full thickness of dysplastic urothelium in 31 (96.9\%) and 29 (90.6\%) cases, respectively (Fig. 3,4). Remaining cases were completely negative.

CD44 and CD66 were completely negative in 29 $(90.6 \%)$ and $31(96.9 \%)$ cases, respectively.

There were no differences in expression pattern between the cases of solitary CIS and CIS associated with the invasive neoplasm.

\section{Non-neoplastic urothelium}

All the studied markers of CSC were also positive in the vast majority of non-neoplastic urothelium samples. CD24 showed continuous positivity of superficial layer of urothelium in $69(93.2 \%)$ cases. In remaining cases the non-neoplastic urothelium was completely negative. 
Table 3. Association of CD44 and CD66 expression with the histopathological characteristics of urinary bladder UC.

\begin{tabular}{|c|c|c|c|c|c|}
\hline & total number & CD 44 positive & $P$ & CD 66 positive & $P$ \\
\hline Grade (WHO 1973) & & & 0.8428 & & 0.2030 \\
\hline Total (UC) & 112 & $73(65.2 \%)$ & & $42(37.5 \%)$ & \\
\hline Grade 1 & 7 & $5(71.4 \%)$ & & $4(57.1 \%)$ & \\
\hline Grade 2 & 30 & $21(70.0 \%)$ & & $14(46.7 \%)$ & \\
\hline Grade 3 & 75 & $48(64.0 \%)$ & & $24(32.0 \%)$ & \\
\hline Grade (WHO 2016) & & & 0.5199 & & 0.1403 \\
\hline Total (UC) & 112 & $73(65.2 \%)$ & & $42(37.5 \%)$ & \\
\hline Low-grade & 35 & $25(71.4 \%)$ & & $17(48.6 \%)$ & \\
\hline High-grade & 77 & $49(63.6 \%)$ & & $25(32.5 \%)$ & \\
\hline Stage classification & & & 0.2048 & & 0.2439 \\
\hline Total (UC) & 112 & $73(65.2 \%)$ & & $42(37.5 \%)$ & \\
\hline $\mathrm{Ta}$ & 37 & $26(70.3 \%)$ & & $18(48.6 \%)$ & \\
\hline $\mathrm{T} 1$ & 16 & $6(37.5 \%)$ & & $4(25.0 \%)$ & \\
\hline $\mathrm{T} 2$ & 16 & $11(68.8 \%)$ & & $7(43.8 \%)$ & \\
\hline $\mathrm{T} 3$ & 26 & $18(69.2 \%)$ & & $9(34.6 \%)$ & \\
\hline $\mathrm{T} 4$ & 17 & $12(70.6 \%)$ & & $4(23.5 \%)$ & \\
\hline Depth of invasion & & & 0.3283 & & 0.3277 \\
\hline Total (UC) & 112 & $73(65.2 \%)$ & & $42(37.5 \%)$ & \\
\hline Non-muscle-invasive $(\mathrm{Ta}+\mathrm{T} 1)$ & 53 & $32(60.4 \%)$ & & $22(41.5 \%)$ & \\
\hline Muscle-invasive (T2+T3+T4) & 59 & $41(69.5 \%)$ & & $20(33.9 \%)$ & \\
\hline Lymph node status & & & 0.7748 & & 0.5398 \\
\hline Total (invasive UC) & 75 & $47(62.7 \%)$ & & $24(32.0 \%)$ & \\
\hline No & 60 & $37(61.7 \%)$ & & $18(30.0 \%)$ & \\
\hline $\mathrm{N} 1+\mathrm{N} 2$ & 15 & $10(66.7 \%)$ & & $2(13.3 \%)$ & \\
\hline
\end{tabular}

UC - Urothelial Carcinoma

CD44 was expressed diffusely in basal layer of urothelium in all cases of cases.

Expression of CD66 and CD 133 was more variable. CD66 showed positivity in 53 (71.6\%) samples of normal urothelium - superficial layer of urothelium was in the pattern as in the case of CD24 positive in 41 cases (77.4\%) and in remaining cases there was observed only focal positivity but of full thickness of urothelium. CD133 was positive in 25 (33.8\%) cases in full thickness of urothelium.

\section{DISCUSSION}

Cancer stem cells (CSC) are proposed as a small subgroup of tumor cells with the ability to self-renew and initiate carcinogenesis by differentiating into heterogeneous tumor cell populations ${ }^{7}$. Moreover, CSC have been shown to be resistant to oncological therapy which represents one of the explanations for recurrence and metastasing of tumors even after therapy ${ }^{16}$. Thus, the identification of CSC in neoplastic tissues could be of help in developing targeted therapy for more efficient cancer treatment in the future.
The existence of CSC has been proven in various solid tumors including urothelial carcinomas ${ }^{3,7,8}$. Several potential CSC markers were identified in in vitro studies by functional analyses (e.g. cell migration, colony formation, sphere formation) and in vivo experiments with xenografts using both cell cultures and human tumor tissue ${ }^{5}$. Only a few studies investigated the identification of CSC directly in tissue sections using immunohistochemistry and analyzed the association of these markers expression with histopathological and prognostic factors in urinary bladder urothelial carcinoma and its precursor lesions $\mathrm{s}^{7,11,13}$.

While CSC are considered as a small subpopulation of cells in tumor mass, our findings showed that all the studied markers were expressed in urothelial tumors more widely than in a limited subpopulation of cells, and in some cases the positivity was even diffuse. Moreover, all the markers were also positive in non-neoplastic urothelium. Therefore, these molecules do not represent exclusive CSC markers, and thus they do not seem to be suitable for CSC detection in UC tissue sections.

However, expression of markers CD24 and CD133 correlated with dedifferentiation of the tumors (i.e. with higher grade) and also with the presence and depth of 


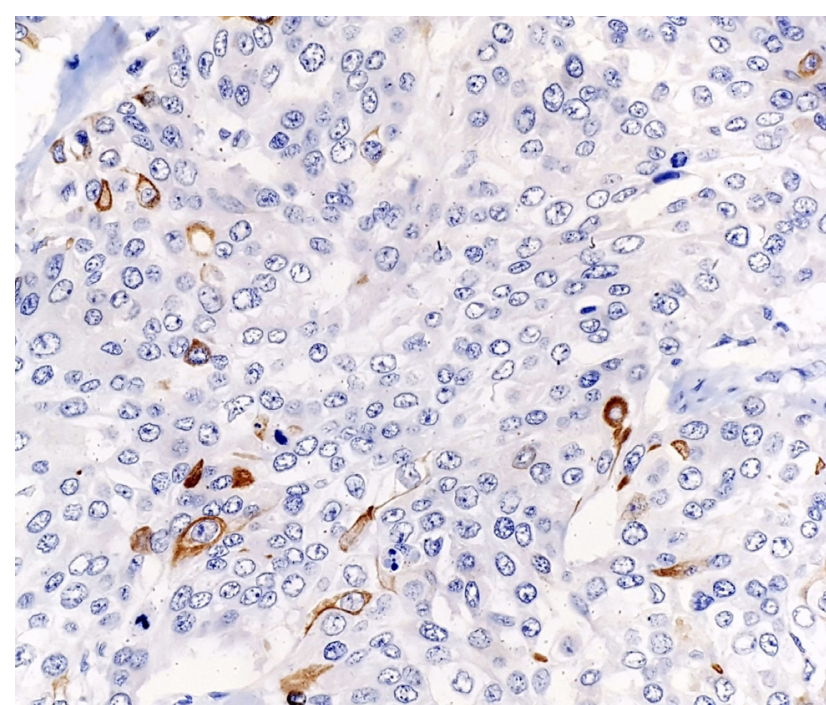

Fig. 1. Focal positivity of CD133 in high-grade urothelial carcinoma (magnification $\mathrm{x} 400$ ).

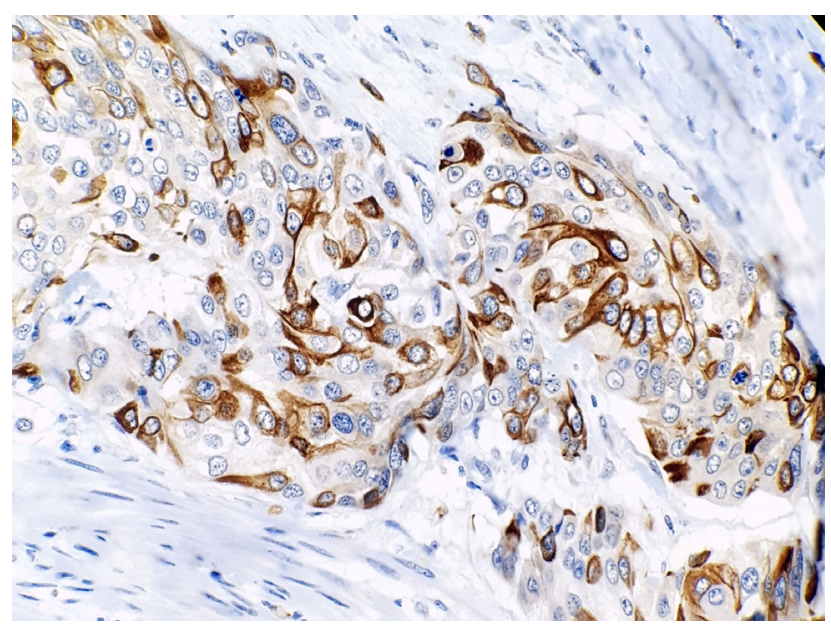

Fig. 2. Diffuse positivity of CD133 in high-grade urothelial carcinoma (magnification $\mathrm{x} 400$ ).

tumor invasion (stage). We also observed expression of CD24 and CD133 in high-grade precancerous lesions (urothelial CIS).

In the work of Liu et al. ${ }^{13}$ the expression of CD24 was associated with increasing tumor grade, but not with stage of studied tumors; however, only non-muscle-invasive bladder urothelial carcinomas were analyzed in that study. In our study including the muscle-invasive carcinomas, the association of CD24 expression was significant both with increasing grade and the stage of the tumors.

In recent work of Farid et al. ${ }^{17}, 50$ cases of urothelial carcinomas including low-grade and high-grade tumors of various stages were analyzed. Similarly to our results, the expression of CD133 and CD24 was associated with increasing tumor grade and depth of the invasion.

As for CD133 expression in UC, there are still controversial results among studies investigating the association between CD133 expression and histopathological factors. The work of Sedaghat et al. ${ }^{18}$ showed decreasing CD 133 expression with increasing invasivity of UC. In our study,

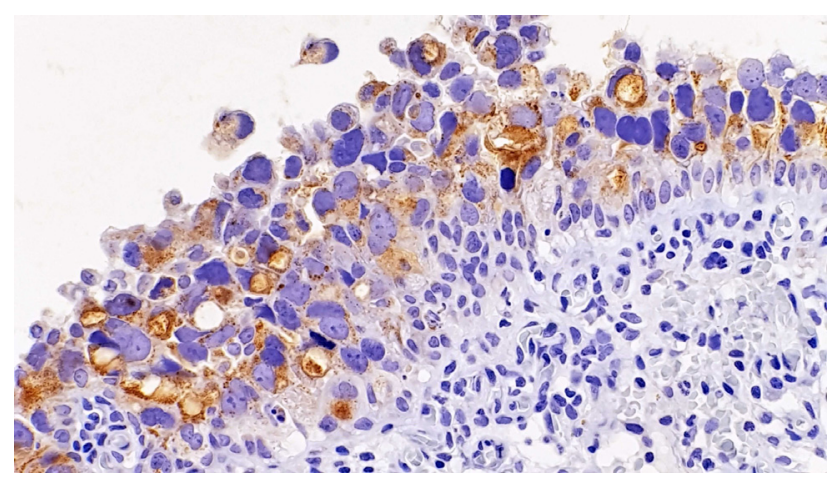

Fig. 3. Expression of CD24 in urothelial carcinoma in situ (magnification $\mathrm{x} 400$ ).

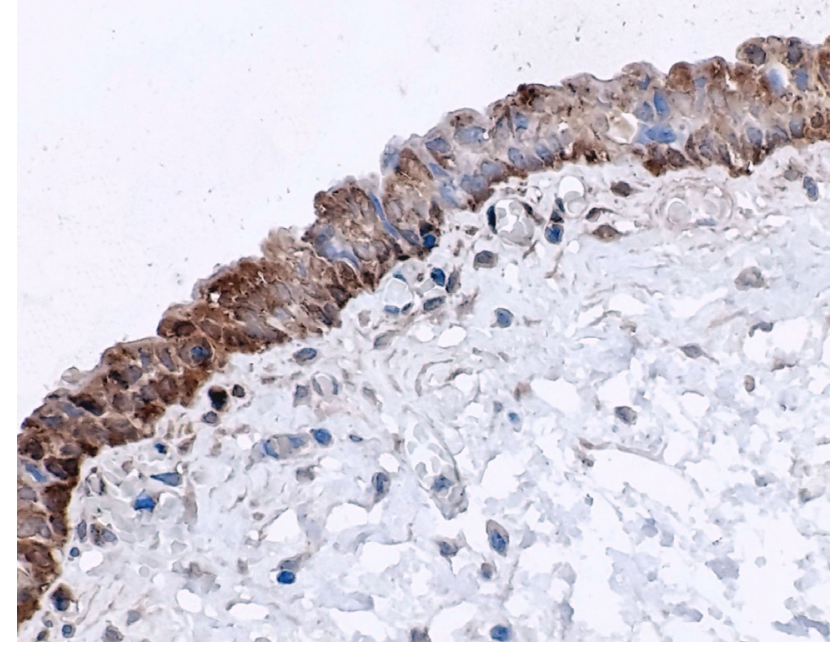

Fig. 4. Expression of CD133 in urothelial carcinoma in situ (magnification $\mathrm{x} 400$ ).

along with the results of Huang et al. ${ }^{10}$, the CD133 expression was associated with increasing tumor stage.

In UC no association of CD44 expression and histopathological parameters was observed in our study, when the whole group of UC with all stages was considered. But, when the Ta and T1 stages only were compared, the expression of CD44 was significantly stronger in the latter and thus it was associated with increased invasivity. A similar result was shown in the study of Desai et al. ${ }^{14}$.

The molecule CD44 was expressed in basal layer of non-neoplastic urothelium and was absent in urothelial CIS. This feature has been used as a diagnostic tool for urothelial CIS (ref. ${ }^{19}$ ). Based on our results, extension of the currently used diagnostic panel (containing antibodies against p53, Ki-67, CK20 and CD44) by anti-CD24 and anti-CD133 should increase the sensitivity of urothelial CIS histologic detection.

Association between CD24 and CD133 expression with tumor grade and stage was shown also in studies of solid tumors other than UC. In colorectal adenocarcino- 
ma, CD24 and CD133 expression correlated with degree of dedifferentiation and depth of invasion, respectively ${ }^{20}$. In pancreatic ductal carcinoma, an increased intensity of CD24 staining was shown when compared to chronic pancreatitis $^{21}$.

\section{CONCLUSION}

In summary, expression of studied molecules CD24, CD44, CD66 and CD133 do not seem to be exclusive markers of CSC in urothelial carcinoma and are not suitable for detecting CSC in tissue sections. However, our study showed that the expression of CD24 and CD133 is significantly related to UC grade and stage, which are both important prognostic indicators. Therefore, the diagnostic detection of expression of these molecules in urothelial lesions might be of potential prognostic value.

Acknowledgments: Special thank to Adrian Cibula, who performed the substantial part of immunohistochemical staining, and to Miroslav Koblizek MD for the help with statistical analysis.

The study was supported by grant GAUK No. 311016.

Author contributions: JH: literature search, data collection and interpretation, figures, manuscript writing; $\mathrm{AB}$ : surgical procedures, patient data analysis; MB: surgical procedures, clinical issues consultation; JZ: data analysis and interpretation, manuscript writing, project supervision.

Conflict of interest statement: The authors state that there are no conflicts of interest regarding the publication of this article. None declared.

\section{REFERENCES}

1. Moch H, Humphrey PA, Ulbright TM, Reuter V. WHO Classification of Tumours of the Urinary System and Male Genital Organs. Lyon, France: International Agency for Research on Cancer; 2016.

2. Siegel RL, Miller KD, Jemal A. Cancer statistics, 2019. CA Cancer J Clin 2019;69:7-34.

3. Visvader JE, Lindeman GJ. Cancer stem cells in solid tumours: ac cumulating evidence and unresolved questions. Nat Rev Cancer 2008;8:755-68.

4. Kreso A, Dick JE. Evolution of the Cancer Stem Cell Model. Cell Stem Cell 2014;14:275-91.

5. Dick JE. Stem cell concepts renew cancer research. Blood 2008;112:4793-807.
6. Bokmen S, Fiser K, Heidenreich O, Vormoor J. Understanding the cancer stem cell. Br J Cancer 2010;103:439-45.

7. Fang D, Kitamura H. Cancer stem cells and epithelial-mesenchymal transition in urothelial carcinoma: Possible pathways and potential therapeutic approaches. Int J Urol 2018;25:7-17.

8. Chan KS, Espinosa I, Chao M, Wong D, Ailles L, Diehn M, Gill H, Presti J Jr, Chang HY, van de Rijn M, Shortliffe L, Weissman IL. Identification, molecular characterization, clinical prognosis, and therapeutic targeting of human bladder tumor-initiating cells. Proc Natl Acad Sci USA 2009;106:14016-21.

9. Jaggupilli A, Elkord E. Significance of CD44 and CD24 as cancer stem cell markers: An enduring ambiguity. Clin Dev Immunol 2012;2012:708036. doi: 10.1155/2012/708036

10. Huang $P$, Watanabe $M$, Kaku H, Ueki $H$, Noguchi $H$, Sugimoto M, Hirata T, Yamada H, Takei K, Zheng S, Xu K, Nasu Y, Fujii Y, Liu $\mathrm{C}$, Kumon $\mathrm{H}$. Cancer stem cell-like characteristics of a CD133+ subpopulation in the J82 human bladder cancer cell line. Mol Clin Oncol 2013;1:180-4.

11. Hofner T, Macher-Goeppinger S, Klein C, Schillert A, Eisen C, Wagner S, Rigo-Watermeier T, Baccelli I, Vogel V, Trumpp A, Sprick MR. Expression and prognostic significance of cancer stem cell markers CD24 and CD44 in urothelial bladder cancer xenografts and patients undergoing radical cystectomy. Urol Oncol 2014;32:678-86.

12. Li Y, Lin K, Yang Z, Han N, Quan X, Guo X, Li C. Bladder cancer stem cells: clonal origin and therapeutic perspectives. Oncotarget 2017;8:66668-79.

13. Liu C, Zheng S, Shen H, Xu K, Chen J, Li H, Xu Y, Xu A, Chen B, Kaku $H$, Nasu $Y$, Kumon $H$, Huang $P$, Watanabe M. Clinical significance of CD24 as a predictor of bladder cancer recurrence. Oncol Lett 2013;6:96-100.

14. Desai S, Lim SD, Jimenez RE, Chun T, Keane TE, McKenney JK, ZavalaPompa A, Cohen C, Young RH, Amin MB. Relationship of cytokeratin 20 and CD44 protein expression with WHO/ISUP grade in pTa and pT1 papillary urothelial neoplasia. Mod Pathol 2000;13:1315-23.

15. Brierley JD, Gospodarowicz MK, Wittekind C. TNM Classification of Malignant Tumours, 8th Edition. Oxford: Wiley-Blackwell; 2016.

16. Soltanian S, Matin MM. Cancer stem cells and cancer therapy. Tumour Biol 2011;32:425-40.

17. Farid RM, Sammour SA, Shehab EIDin ZA, Salman MI, Omran TI. Expression of CD133 and CD24 and their different phenotypes in urinary bladder carcinoma. Cancer Manag Res 2019;11:4677-90.

18. Sedaghat S, Gheytanchi E, Asgari M, Roudi R, Keymoosi H, Madjd Z. Expression of Cancer Stem Cell Markers OCT4 and CD133 in Transitional Cell Carcinomas. Appl Immunohistochem Mol Morphol 2017;25:196-202.

19. Lopez-Beltran A, Montironi R, Vidal A, Scarpelli M, Cheng L. Urothelial dysplasia of the bladder: diagnostic features and clinical significance. Anal Quant Cytopathol Histpathol 2013;35:121-9.

20. Choi D, Lee HW, Hur KY, Kim JJ, Park GS, Jang SH, Song YS, Jang KS, Paik SS. Cancer stem cell markers CD133 and CD24 correlate with invasiveness and differentiation in colorectal adenocarcinoma. World J Gastroenterol 2009;15:2258-64.

21. Durko L, Wlodarski W, Stasikowska-Kanicka O, WagrowskaDanilewicz M, Danilewicz M, Hogendorf P, Strzelczyk J, MaleckaPanas E. Expression and Clinical Significance of Cancer Stem Cell Markers CD24, CD44, and CD133 in Pancreatic Ductal Adenocarcinoma and Chronic Pancreatitis. Dis Markers 2017;2017:3276806. 